\title{
Stage IVB Thymoma AJCC v8
}

National Cancer Institute

\section{Source}

National Cancer Institute. Stage IVB Thymoma A/CC v8. NCI Thesaurus. Code C136356.

Stage IVB includes: (Any T, N2, M0, M1a); (Any T, Any N, M1b). N2: Metastasis in deep intrathoracic or cervical lymph nodes. M0: No distant metastasis. M1a: Separate pleural or pericardial nodule(s). M1b: Pulmonary intraparenchymal nodule or distant organ metastasis. (AJCC 8th ed.) 